\title{
A Low-Power and Low Cost Smart Streetlight System Based on Internet of Things Technology
}

\section{Jingjing Zhang}

China University of Geosciences Beijing https://orcid.org/0000-0003-1982-5523

Weihua Zeng ( $\nabla$ zwh@cugb.edu.cn )

China University of Geosciences Beijing https://orcid.org/0000-0003-1477-4675

\section{Shengli Hou}

China University of Geosciences Beijing

\section{Yuqi Chen}

China University of Geosciences Beijing

\section{Linyan Guo}

China University of Geosciences Beijing

Yanxing Li

China University of Geosciences Beijing

\section{Research Article}

Keywords: Smart streetlight, Low power, Energy saving, LoRa, NB-loT

Posted Date: June 21st, 2021

DOl: https://doi.org/10.21203/rs.3.rs-613714/v1

License: (c) (i) This work is licensed under a Creative Commons Attribution 4.0 International License. Read Full License

Version of Record: A version of this preprint was published at Telecommunication Systems on November 3rd, 2021. See the published version at https://doi.org/10.1007/s11235-021-00847-1. 


\title{
A low-power and low cost smart streetlight system based on Internet of Things technology
}

\author{
Jing-jing Zhang ${ }^{1}$. Wei-hua Zeng ${ }^{1}$ • Sheng-li Hou ${ }^{1}$ • Yu-qi Chen ${ }^{1}$ • Lin-yan Guo ${ }^{1}$. \\ Yan-xing $\mathrm{Li}^{1,2}$
}

Received: date / Accepted: date

\begin{abstract}
Street lighting, as the most essential and universal component of the urban lighting system, accounts for a large portion of public electricity usage. Therefore, improving street lamps working efficiency is vital for energy savings. This paper demonstrates the design of a smart street lighting system supported by the combination of NB-IoT and LoRa communication technology. By adopting an optimized street lamp control algorithm, the system can realize the automatic control of street lights according to the realtime traffic flow information. This system has been installed on Luyang Avenue, Lucheng City, Shanxi Province, China in May 2019. It managed to reduce the electricity consumption in this region significantly in June 2019, which was $18 \%$ lower than that in April 2019 and 19.7\% lower than that in June 2018. By illustrating the unique advantages of this system in energy savings and cost reduction, this paper displays its potential for further application in the construction of smart cities on a large scale.
\end{abstract}

Jing-jing Zhang

2010200028@cugb.edu.cn

Wei-hua Zeng

zwh@cugb.edu.cn

Sheng-li Hou

slhou@cugb.edu.cn

Yu-qi Chen

2110200010@cugb.edu.cn

Lin-yan Guo

guoly@cugb.edu.cn

Yan-xing Li

66369280@qq.com

${ }^{1}$ School of Geophysics and Information Technology, China University of Geosciences, Beijing 100083, China

2 Coal Geological Exploration Surveying \& Mapping Institute of Shanxi Province, China
Keywords Smart streetlight · Low power · Energy saving · LoRa $\cdot$ NB-IoT

\section{Introduction}

As a common source of lighting, street lamp is an important part of urban road lighting system. With the expansion of urban roads and the growth of people's daily travel demand, the number of street lamps in each area increases at a linear rate $[1,2]$. The quantity of urban street lamps in China has risen from just over 10 million to nearly 30 million from 2004 to 2019 . The long-term power supply required by street lamps results in about $35 \%$ of the electricity being dissipated every year [3]. Therefore, in order to improve the energy utilization rate and reduce the waste of electricity in urban road lighting system, appropriate control methods must be taken to improve the work efficiency of street lamps and further promote the scientific management and green energy saving of urban lighting system [4].

Traditional street lamp control methods such as manual, induction, and timing control are low in intelligence and cannot meet the requires of modern urban construction. With the rapid development of communication and microelectronics technology, intelligent streetlight system with Internet of Things [5-13] has gradually become the most potential research field, such as using wireless network technology like ZigBee to monitor the status of street lamps and to detect traffic flow and environment.

In terms of monitoring the working status of street lamps, Fabio Leccese et al. [14,15] designed a street lighting system, which obtained with ZigBee to collect the parameter information of street lamps and verify the working state of the street lamps. In 2016, Francisco José et al.[16] proposed an intelligent street lamp management system based on wireless communication and DALI protocol, which could not 
only realize real-time monitoring of running state of the street lamps, but also developed a mobile device information and fault location application program SCADA. In terms of road environmental perception, Philip Tobianto Daely et al.[17] designed an intelligent streetlight system on account of Web integrated management in 2017, using intelligent LED street lamps with weather-sensing function. In terms of the monitoring of traffic flow, Pilar Elejoste et al.[18] proposed a street lamp control system based on wireless communication and LED technology in 2013, which could detect the flow of vehicle flow and the running condition of the street lamps. However, its complex system design and man-sized hardware circuit implementation made it not suitable for largescale application.

In other aspects of street lamp control, many scholars have made great achievements in some research directions such as artificial intelligence, big data model optimization . Among them, as a new algorithm, Artificial Neural Network (ANN) is one of the heat spots of many scholars[19, 20]. Based on the analysis of the existing control methods of street lighting, the idea of "energy on demand" on account of ANN modeling method was put forward by S.Pizzuti et al. in 2013[21]. Ulteriorly, Juan F. De Paz et al.[22] proposed an intelligent urban lighting control system in 2016, which integrated advanced technologies such as ANN, Expectation Maximization (EM) algorithm. In 2019, Prabu Mohandas et al.[23] also proposed an energy-saving intelligent street lighting system based on ANN, which reduced the power dissipation by $13.5 \%$. Besides, in terms of Wireless Sensor Network (WSN), Soleda Decolar[24] proposed an intelligent streetlight control system in 2014, which based on adaptive behavior rules. The control equipment on the street lamp post could dynamically adapt to the presence of pedestrians and vehicles around it. In 2019, He Junjian et al.[25] further used WSN technology to obtain road environment parameters. The algorithm implementation of the intelligent streetlight control system based on artificial intelligence technology is relatively complex, and the requirements for hardware circuit components are relatively high, which make it not easy to large-scale field applications.

At present, although the pilot project of the intelligent street lamp has been established in some Chinese cities [26], it has not yet formed a scale and the most street lamp control strategies do not incorporate real-time traffic flow information. Therefore, on the basis of analyzing the existing technology, an intelligent streetlight system with low power consumption and low construction cost is designed in this paper. The proposed system adopted low-power hardware circuit components and communication technology and combined with a streamlined street lamp control strategy, which could intelligently adjust the lighting time of the street lamp according to the traffic flow. At the same time, the cloud server side can monitor the working state of street lamps in real time through Narrow Band Internet of Things so as to facilitate the timely handling of street lamps when they work failed, which realizes the intelligent management of urban streetlight under the premise of energy saving and environment protection. By analyzing the use of electricity consumption of the application area before and after the usage of proposed system, it has greatly improved the energysaving effect of street lamps, where the electricity consumption in June 2019 was $18 \%$ lower than that in April 2019 and $19.7 \%$ lower than that in June 2018.

The rest of the paper is structured as follows. The overall structure design of the intelligent streetlight system and the functions of each module are introduced in Sect. 2, the hardware circuit design of the motion detector unit and streetlight controller are briefly discussed in Sect. 3, and Sect. 4 depicts the software design of the system. The practice application effect and analysis of this system are illustrated in Sect. 5, and the conclusion is summarized in Sect. 6.

\section{The overall architecture of the system}

The structure of the smart streetlight system proposed in this paper consists of three main parts: Motion Detector Unit (MDU), Streetlight Controller and Cloud Server. The architecture of the whole system is shown in Figure 1.

MDU and Streetlight Controller are the control core of the smart streetlight system, which communicate through the LoRa network. According to the data transmission distance of LoRa communication, street lamps within the same LoRa communication range are divided into a street lamp group. Each street lamp group consists of a street lamp with MDU and about ten street lamps members. The spacing between each street lamp is approximately 50 meters. MDU monitors the traffic flow information based on the principle of Doppler velocimetry and combines the optimized streetlight control algorithm to calculate the street lamp controlling command, which is transmitted it to the Streetlight Controller through the LoRa network to realize the dynamic control of the street lamps. Besides, the MDU is also equipped with an NB-IoT module through which the working status of MDU can be uploaded to the Cloud Server.

The Streetlight Controller receives the control demand which sent by MDU through LoRa network to executes the corresponding command to realize the control of the street lamps on and off. In addition, it can also upload the status messages of street lamps such as voltage, current to Cloud Server through NB-IoT network, which is more convenient for staffs to realize the monitoring of the working state of the street lamps.

Cloud Server mainly includes three functional modules: Account management module, Device management module and Dispatch service module, which is implemented on 


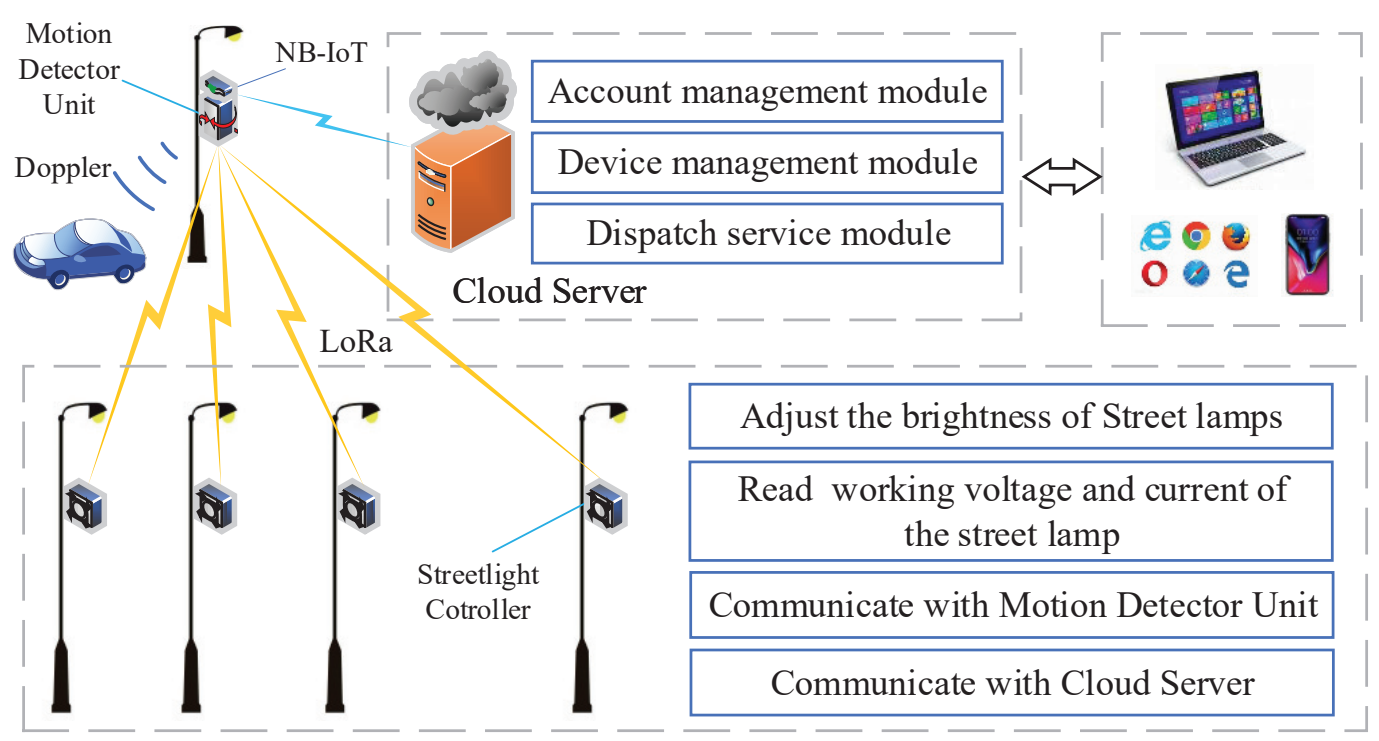

Fig. 1 Functional structure of the system

computer, mobile phone and other terminals. The Device management module can monitor whether MDU and street lamp work normally. Dispatch service module means that when the Cloud Server detects that the working status information of the street lamp is abnormal, the damage informations of the street lamp is printed to the maintenance workers, so as to realize the effective management of the street lamp.

\section{Hardware circuit design of the smart streetlight system}

\subsection{The hardware design of MDU}

\subsubsection{Principle of Doppler velocity measurement}

The Doppler effect of electromagnetic wave points out that when the object and the electromagnetic wave source are in relative motion, the reflected electromagnetic wave frequency $f_{r}$ will change. The change between $f_{r}$ and the emitted wave frequency $f_{s}$ is the Doppler frequency $f_{d}$. When the target object is moving toward the emission source, due to the Doppler effect, the frequency of the electromagnetic wave signal returned from the target will be higher than the frequency of the emission signal. The calculation formula of Doppler frequency is as follows:

$f_{d}=f_{s}-f_{r}=2 v \frac{f_{s}}{c} \cos \theta$

where $v$ is the velocity of moving object, $c$ is the speed of light, and $\theta$ is the angle between the direction of movement of the object and the normal of the detection surface of the detector. It can be seen from the calculation formula that the Doppler frequency is proportional to the relative speed of the electromagnetic wave emitting source and the moving target. Therefore, when the Doppler frequency of the target is measured, the velocity of the target relative to the electromagnetic wave emission source can be obtained by using this calculation formula.

\subsubsection{Dopplor Motion Detection Unit MDU6220}

The X-band Doppler motion detector unit MDU6220 of SMS is used to detect the movement of vehicle.Figure. 2 shows the structure of MDU6220.

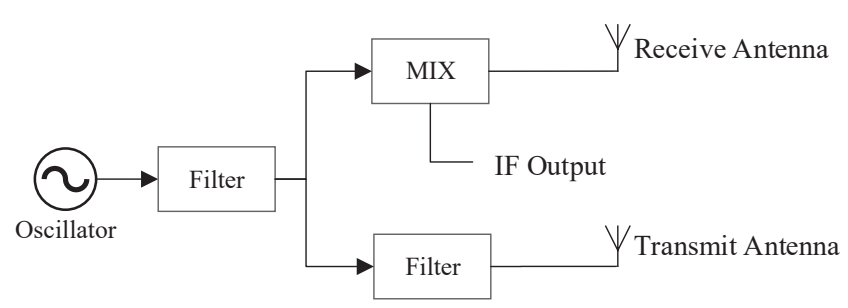

Fig. 2 MDU6220 Structure diagram

MDU6220 is an integrated microwave transmitter and receiver module. It contains a microwave transistor oscillator inside, which can provide a signal with stable frequency and amplitude at unit operating frequency. This signal is filtered to reduce harmonics and spurious signals, and then divided into two signals of approximately equal amplitude. One signal is further filtered and fed into the transmitting antenna and the other signal is fed into the local oscillator input. When the transmitted signal encounters an object in the process of traveling, the signal reflected back will be collected by the receiving antenna and coupled with the Radio 
Frequency input of the balanced mixer. The coupled signal is compared with the transmitted signal to obtain the Doppler frequency, and the subsequent signal processing is performed on the Intermediate Frequency (IF) output of the module. It is worth noting that the balanced mixer configuration provides superior matching and lower conversion loss compared with single-ended mixers, which improves the sensitivity of MDU6220 and enhances its capture capability while reducing false detections.

The amplitude of the IF output signal is proportional to the volume of the measured object and its distance from the MDU6220, and the frequency is proportional to the speed of the detected target. In addition, the MDU6220 module can generate two orthogonal pulse signals to detect the direction of movement of the target object by detecting its forward or lag phase. By designing conditioning and screening circuits to process the IF output signal, the traveling direction and speed of the vehicles are judged, which lays the hardware foundation for the system software implementation.

\subsubsection{Hardware circuit design of $M D U$}

The hardware circuit design block diagram of the MDU is shown in Figure 3. In order to reduce the power consumption of the MDU itself, the main control MCU adopts 32-bit chip STM32L151 based on the Cortex-M3 architecture of ST. The ultra-low power dissipation mode of the chip can achieve low depletion while also meeting the requires of design without reducing system performance.

The IF signal is counted by the MCU counter after lowpass filtering, programmable amplification and voltage discrimination. When the frequency of the measured signal is larger than the preset value, it is determined that a vehicle has entered the detection area. The vehicle information detected by MCU will generate streetlight control commands through an optimized streetlight algorithm. Then it is transmitted to the Streetlight Controller through the LoRa network, which realizes the on and off control of the streetlight according to the received command. Moreover, the MDU is also equipped with NB-IoT module, through which the Cloud Server can monitor its working state.

Considering that other interference factors such as leaf fluttering caused by wind or running of small animals may lead to the misjudgment of MDU and lighting up street lamps will result in further power dissipation, the digital potentiometer and amplitude discrimination are used in the hardware circuit design to adjust the feedback resistance of the amplifier circuit to realize the programmable processing of the output signal of MDU. Only when the volume of the detected object is larger than a certain value can the counting system be triggered effectively. At the same time, the lowfrequency disturbance is filtered through software, which avoids misjudgment caused by interference and makes the result more accurate.

\subsection{Hardware circuit design of Streetlight Controller}

The number of Streetlight Controllers is much higher than that of MDU in practical applications. Therefore, the power consumption of the Streetlight Controller itself has a great influence on the power consumption of the entire system. The MCU of the Streetlight Controller uses STM32L151 microcontroller as well as MDU, which enables it to reduce power consumption while maintaining its processing speed. Figure 4 shows the hardware circuit design diagram of the Streetlight Controller.

The command to control street lamps that the MDU transmits to the MCU of the Streetlight Controller through LoRa module is the foundation to realize the control of street lamps. Besides, the Streetlight Controller is equipped with sensors to detect the voltage and current of street lamp, which can be uploaded to the cloud server through the NB-IoT network so as to realize the real-time monitoring of the working state of the street lamps.

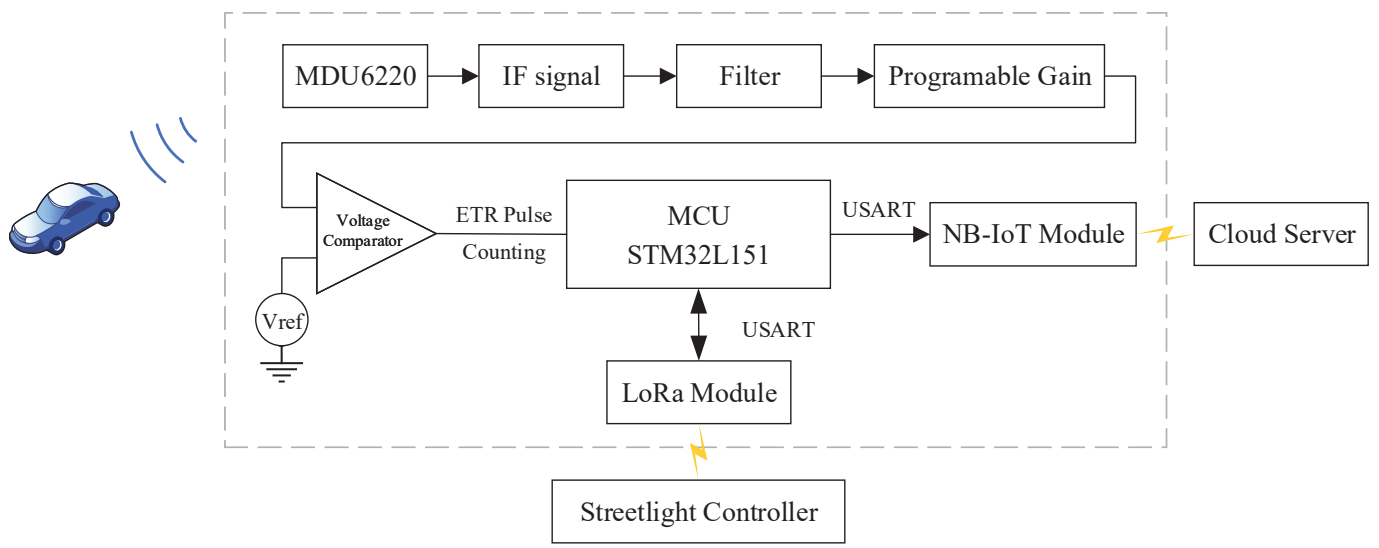

Fig. 3 Hardware circuit design of MDU 


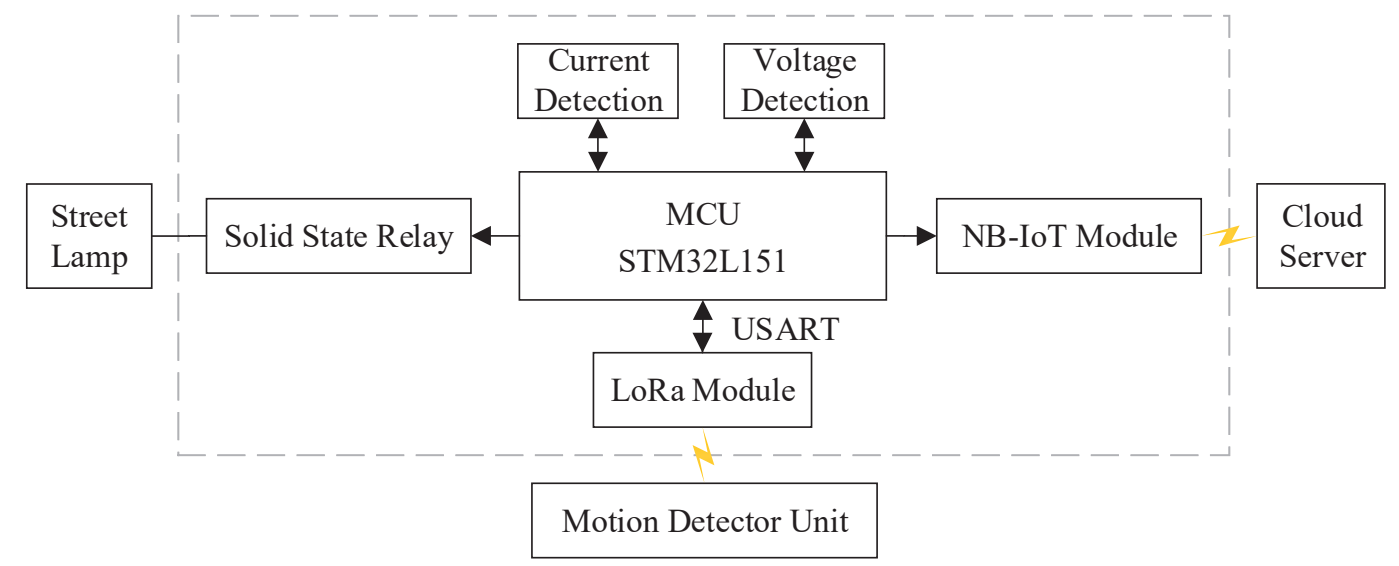

Fig. 4 Hardware circuit design of Streetlight Controller

\section{Software design of the smart streetlight system}

\subsection{System communications design}

\subsubsection{Communication between MDU and Streetlight Controller}

The MDU and Streetlight Controller are 50m to several kilometers apart in actual application. It would be complicated and expensive to use long distance wired connections. By comparing the wireless communication modes commonly used in streetlight control shown in Table. 1, combined with the low-power design goal of the system, LoRa is selected to implement the communication between street lamps.

LoRa is a low-power Local Area Network (LAN) wireless communication technology introduced by Semtech in 2013. It can achieve the purpose of long-distance transmission under the condition of low power consumption, which make it wide use in many fields such as smart city, smart home and so on [27-29]. Therefore, the wireless serial port module E32(433T30S) from Chengdu YIBEST Electronic Technology Company is selected as the core part of LoRa communication in the system design.

In addition to the realization of long-distance transmission under the condition of low power consumption, this part has strong anti-interference ability and suppression ability to the same frequency interference and various noises. At the same time, it can also realize the function of sending and receiving data by broadcasting. Therefore, within the effec- tive communication range of LoRa, each street lamp only needs to communicate with the street lamp equipped with MDU. The Streetlight Controller under the control of the same MDU chooses the same frequency point and distinct communication address. Depending on this, the MDU can use the broadcast mode to send control demands to each Streetlight Controller through addressing, so as to achieve the effect of turning on and off the street lamps in turn.

\subsubsection{Communication between MDU, Streetlight Controller and Cloud Server}

NB-IoT is a low-power LAN based on authorized frequency band. It is widely used in smart cities, public utilities, logistics and other industries due to its excellent characteristics, such as wide coverage, low construction cost and no interference between equipment in the same base station [30].

Therefore, NB-IoT is adopted in this paper to implement the communication between Cloud Server, MDU and Streetlight Controller. On the one hand, it can monitor the working state of MDU to ensure the normal working of the whole system; on the other hand, the operating state of the street lamps can be uploaded to the Cloud Server by the Streetlight Controller through NB-IoT network. Once an error warning is found in the system, the fault information will be pushed to the maintenance workers via mobile phone, which can make sure they handle the abnormal street lamp in time.

Table 1 Wireless commuunication modes commonly used in streetlight

\begin{tabular}{lccc}
\hline Category & LoRa & Sigfox & ZigBee \\
\hline Coverage Area & $10 \mathrm{~km}$ & $13 \mathrm{~km}$ & Short Distance \\
Validity of Battery & More than 10 years & $8-10$ years & About 1-2 years \\
Date Rate & $50 \mathrm{kbits} / \mathrm{s}$ & $100 \mathrm{kbits} / \mathrm{s}$ & $<250 \mathrm{kbps}$ \\
Current Situation & Comparativ maturity & Mature commercial & Comparativ maturity \\
Network Deployment & Isolated network & Coverage Sigfox network & Isolated network \\
\hline
\end{tabular}




\subsection{Algorithm of street lamp controlling}

As the core of system operation, the algorithm implementation of street lamp control is also the focus of system software design. Based on the effective detection distance of MDU to the vehicle, only the monitoring of one-way vehicles is considered in this system. The operation mode of the whole system is that the street lamps are not energized in the daytime. Light up half of the street lamps at night intervals while the other half are on or off depending on the traffic information of the road. Concrete streetlighting control algorithm can be roughly divided into two situations to consider: when the moving object detection unit detects only one vehicle passing within a certain time (such as 30s) and when there are multiple vehicles passing at distinct speeds and distances.

\subsubsection{Control of street lamps when there is only one car passing}

As shown in Figure 5, when the MDU detects only one vehicle passing by within 30s, the control demands are sent to the corresponding Streetlight Controller by LoRa to light the $1-n$ street lamps after time $t_{n}=d / v(n-1)$, where $v$ is the speed of vehicle. Assuming that the speed of the vehicle is constant when passing the road section, the time interval $\mathrm{T}$ required for each Streetlight Controller from lighting to turning off the street lamp is also the same.

\subsubsection{Control of street lamps with unequal speeds and distance of multiple vehicles}

When more than one car entering at varying speeds and unequal distances in a short time is detected by the MDU, Figure 6 shows the related algorithm flow.

When MDU detects that the first vehicle driving at speed $v_{1}$, the time interval to sequentially turn on the $1-n$ street lamps are set to $t_{1}$, and all turn off after the time $T_{1}$. When it is detected that the second vehicle is driving at speed $v_{2}$ at the moment, the time interval to turn on the $1-n$ street lamps in sequence is still $t_{1}$ if $v_{1}>v_{2}$, but turn off after the time $T_{2}\left(T_{1}<T_{2}\right)$; If $v_{1}<v_{2}$, on the premise of judging that the second car can catch up with the first car, set the time interval to turn on the $1-n$ street lamps in sequence as $t_{2}\left(t_{1}>t_{2}\right)$, but turn off after $T_{1}$. The MDU continues to detect whether there is a vehicle, and the value of the vehicle count variable $\mathrm{Cnt}$ is increased by one once a vehicle is monitored. The vehicle count variable Cnt will be cleared and a new round of detection will be restarted When the next vehicle is not detected within 30 s.

\subsection{Software development}

The embedded program of STM32L151 microcontroller is written by using IAR embedded software development tool with high compiling efficiency to realize the detection of moving objects and the control of street lamps.

On the IAR development platform, the STM32Lxx HAL library was used to complete the embedded programming design of MDU and Streetlight Controller. The main func-

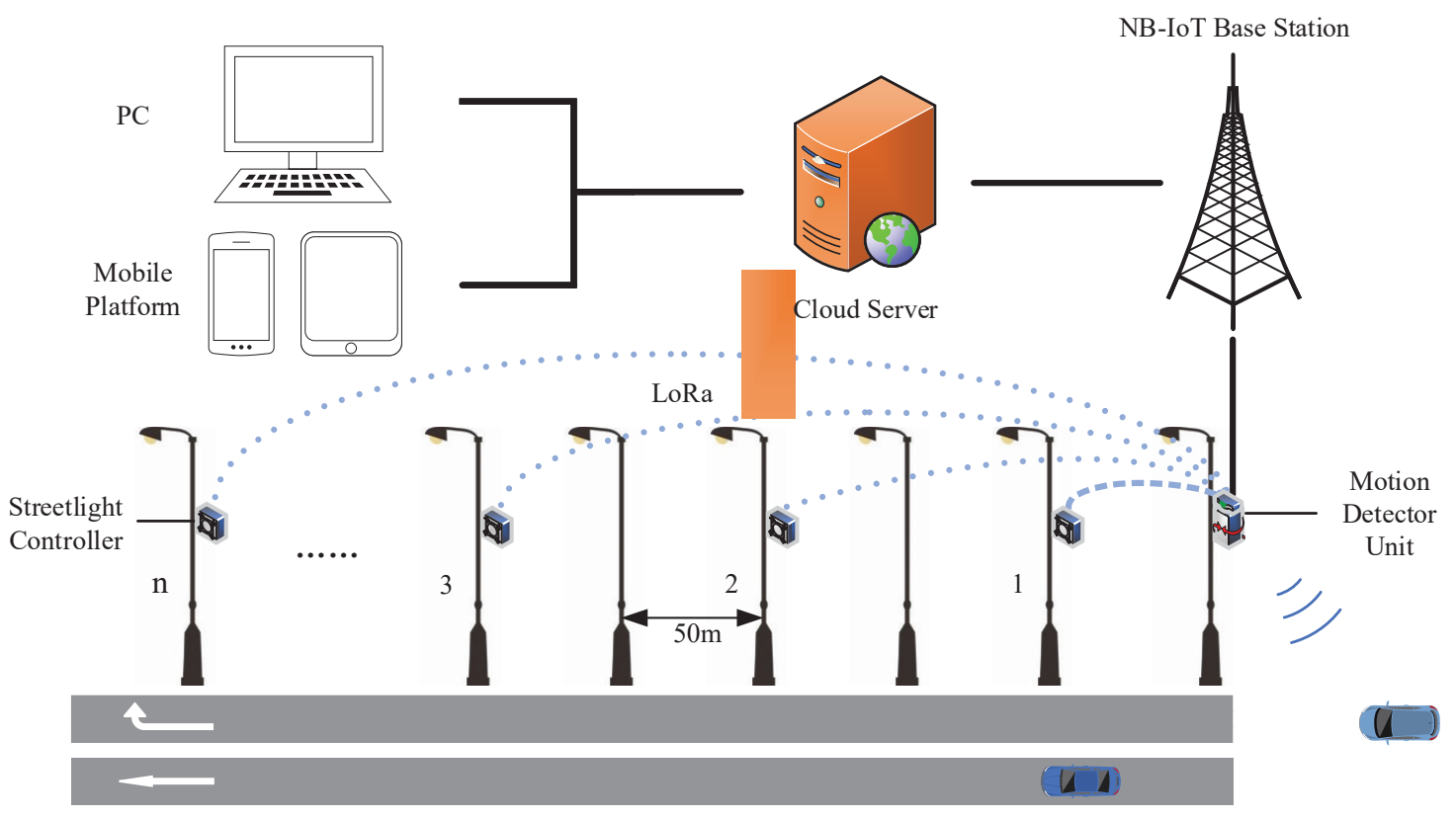

Fig. 5 Schematic scenario 


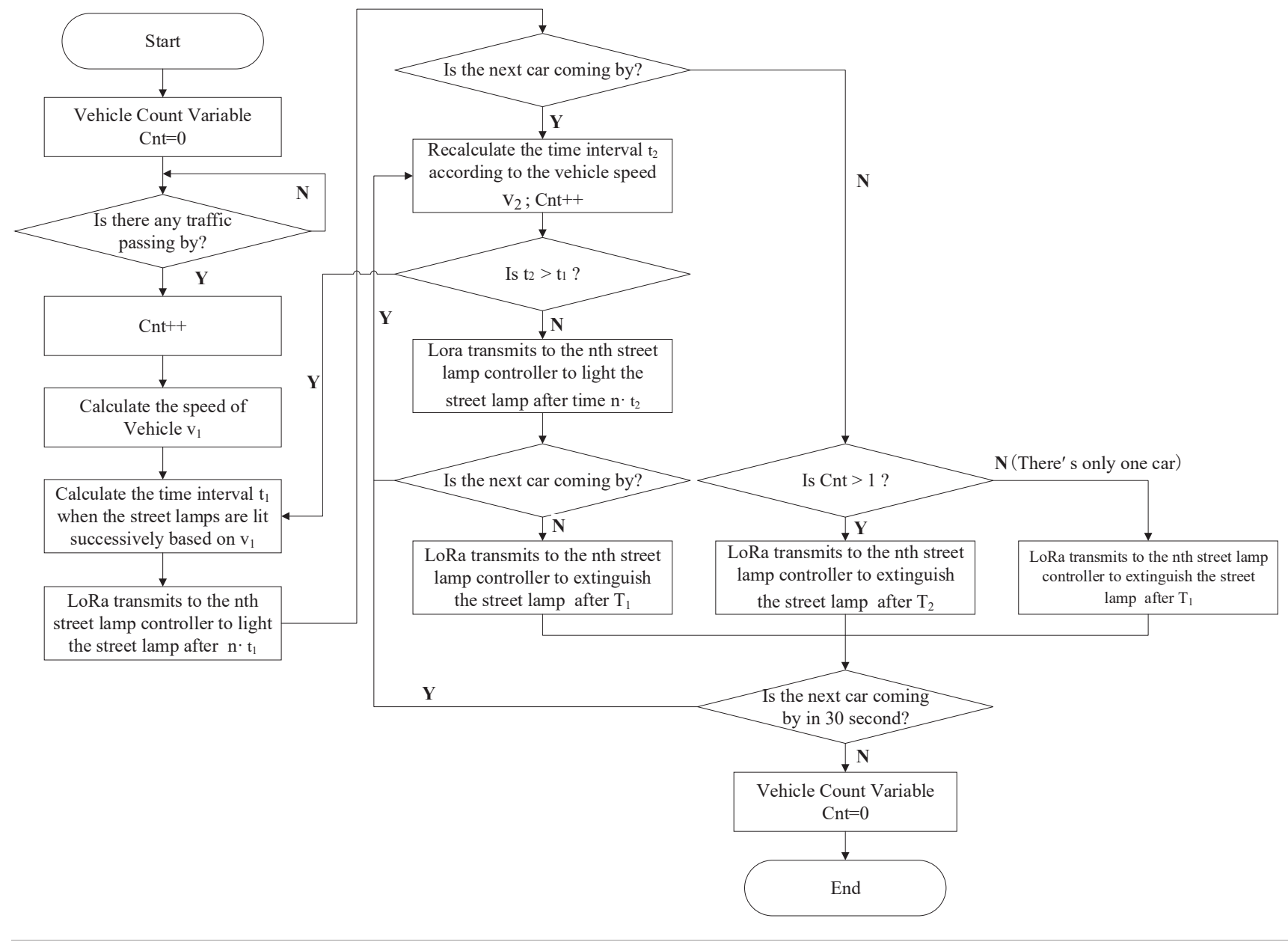

Fig. 6 Algorithm flow of street lamp control for more than one vehicle

tional modules of the program are: hardware initialization module, frequency measurement module, and communication protocol realization module. Among them, the first module includes the initialization of timer, system interrupt and USART serial port and frequency point setting of LoRa module etc. The frequency measurement module is realized by the counter of the CPU, which realizes the pulse counting in time unit through ETR function. The communication protocol implementation module of the Streetlight Controller receives and parses the LoRa serial port packet, so as to control the lights on and off of street lamps.

\section{Field application}

The system has been installed on-site on Luyang Avenue, Lucheng City, Shanxi Province in China. After more than a year field testing, as well as continuous improvement, it has been able to implement the expected results. The field application diagram of Streetlight Controller and MDU are shown in Figure 7 and Figure 8.

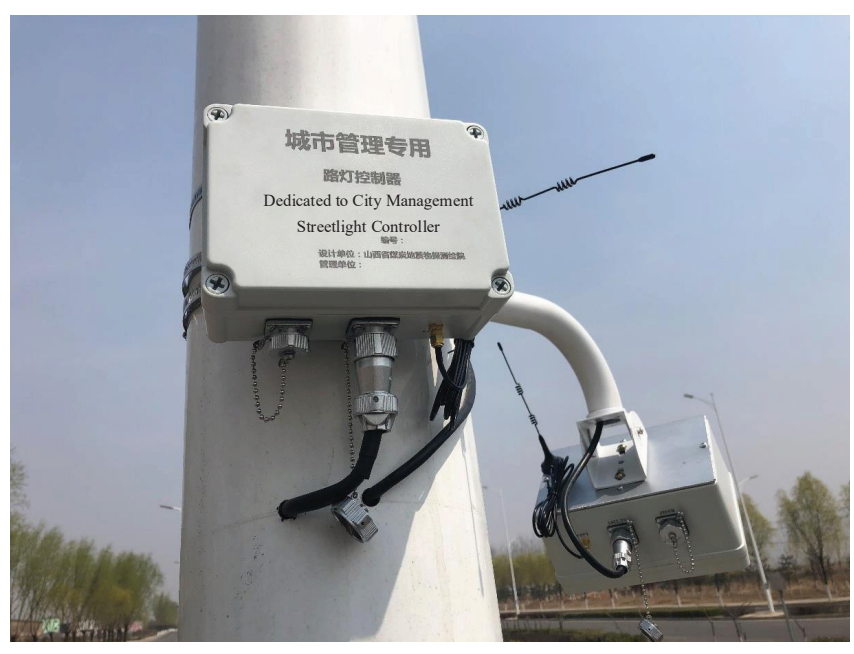

Fig. 7 Streetlight Controller

MDU is installed towards the direction of the oncoming vehicle. The installation angle and internal parameter settings of each MDU are adjustable, which enables it to accurately capture vehicles in four lanes on one side and en- 


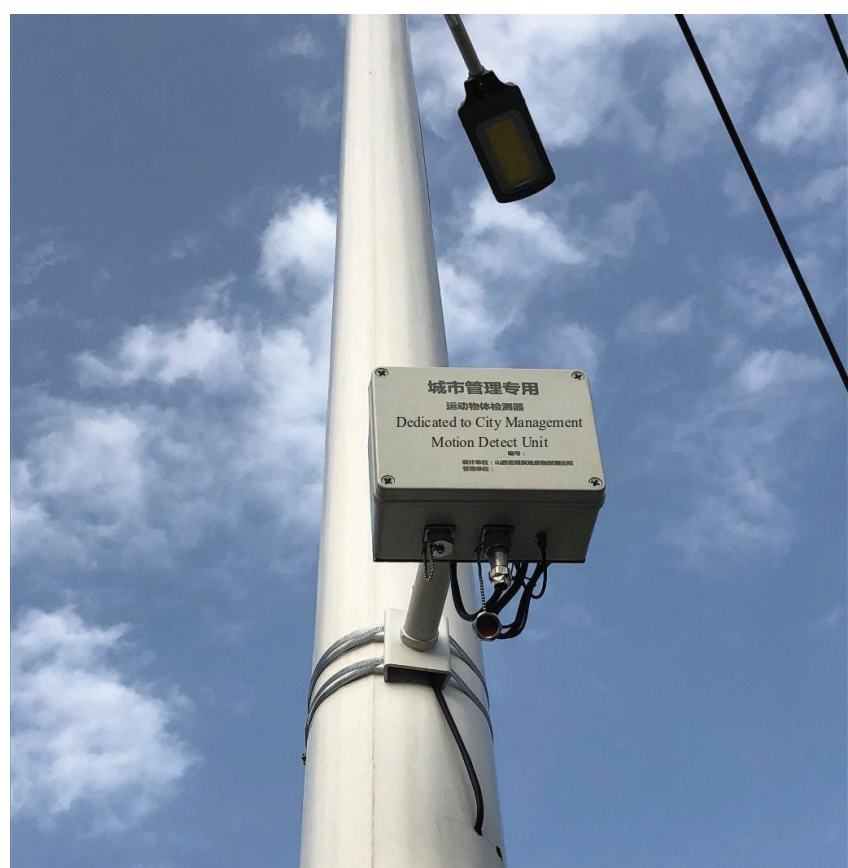

Fig. 8 Motion Detector Unit

sures the precision of control. Adopting an interval control methos, each MDU controls 10 street lamps under normal circumstances (it can be adjusted appropriately according to specific road conditions). A total of 18 MDUs and 165 Streetlight Controllers were installed on Luyang Avenue, accounting for $46 \%$ of the total number of street lamps.

The system was formally put into use in Luyang Avenue in May 2019. The street lamps of Luyang Avenue are controlled by four distribution boxes. The electricity consumption data of the four distribution boxes of Luyang avenue in 2018 and 2019 are shown in Figure 9.

It can be seen that the electricity consumption in June 2019 was 18\% lower than that in April 2019 and 19.7\% lower than that in June 2018 after the intelligent streetlight system was put into use in Luyang Avenue, which significantly reduced the electricity consumption. When MDU and
Streetlight Controller work normally, the average power consumption measured are 9.18mA@ 220V and 10.20mA@ $220 \mathrm{~V}$ respectively, which meets the design goal of low power consumption of the system.

In addition, in order to test the function of reporting faulty street lamp information to the cloud server, the power supply lines of a street lamp is artificially disconnected while the system working normally. The smart street lamp cloud server can detect the abnormal variations of the street lamp working status, and then send the information of the street lamp to the maintenance worker to handle the abnormal street lamp in time, which achieves effective management of street lamps and furtherly improves the intelligent level of urban street lamp management.

\section{Conclusion}

An intelligent streetlight system based on Internet of Things technology is put forward in this paper. Based on the hardware circuit of MDU and Streetlight Controller designed by low power consumption microcontroller STM32L151, the proposed system adopts the low-power communication technology of LoRa and NB-IoT, which implements the intelligent control of cities under the premise of low power consumption and construction cost. In addition, the working voltage and current information of street lamps can be monitored through NB-IoT, which can effectively improve the timeliness of street lamp maintenance and management. Practical application results show that the system can achieve $80 \%$ energy saving for a single lamp and about $20 \%$ overall energy saving on the premise of meeting travel stipulates, i.e. lights when the vehicle comes and goes out when the vehicle is away. Its good energy-saving effect, excellent fault detection, low power consumption and cost characteristics beneficially enable it to be applied to the construction of urban road facilities on a large scale which makes it have favourable economic and social benefits.

Luyang Avenue Electricity Consumption Statistics

Unit: Yuan

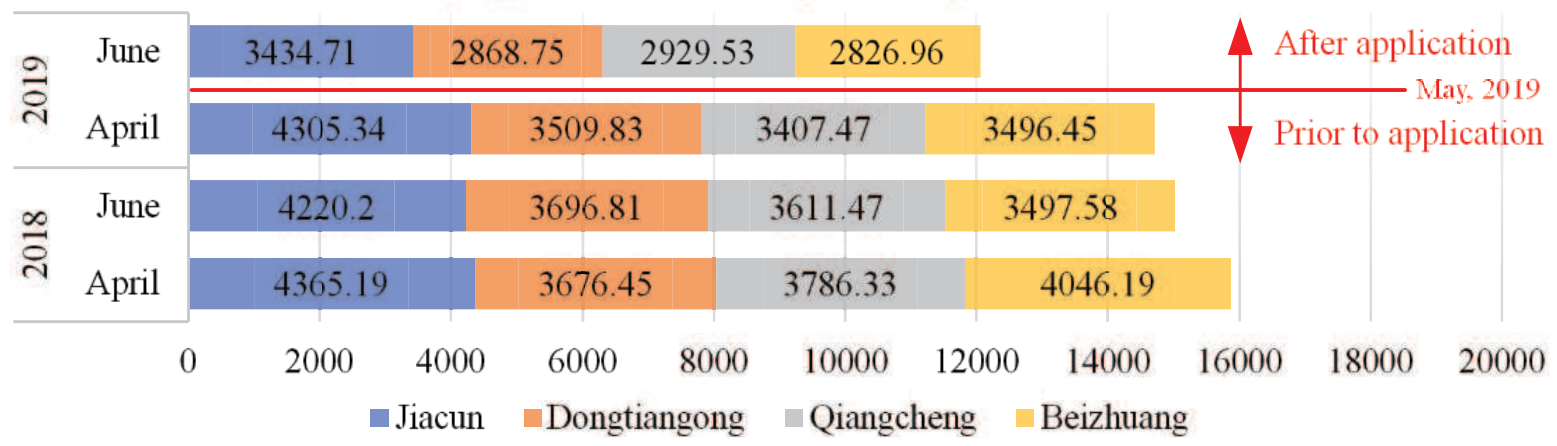

Fig. 9 Luyang Avenue electricity consumption statistics 
Acknowledgements We would like to thank Coal Geological Exploration Surveying \& Mapping Institute of Shanxi Province in China for its strong support in the design and implementation of the project.

\section{Conflict of interest}

On behalf of all authors, the corresponding author states that there is no confict of interest.

\section{References}

1. J. Laufs, H. Borrion, B. Bradford, Sustainable Cities and Society 55, 102023 (2020). DOI 10.1016/j.scs.2020.102023

2. G. Pasolini, P. Toppan, F. Zabini, C.D. Castro, O. Andrisano, Applied Sciences 9(16), 3281 (2019). DOI 10.3390/app9163281

3. F. Lv, Z. Wang, Y. Ding, Y. Li, N. Zhu, Energy Efficiency 9(5), 1037 (2016). DOI 10.1007/s12053-015-9408-5

4. A. Gharaibeh, M.A. Salahuddin, S.J. Hussini, A. Khreishah, I. Khalil, M. Guizani, A. Al-Fuqaha, IEEE Communications Surveys \& Tutorials 19(4), 2456 (2017). DOI 10.1109/COMST.2017.2736886

5. R. Caponetto, G. Dongola, L. Fortuna, N. Riscica, D. Zufacchi. Power consumption reduction in a remote controlled street lighting system (2008)

6. E. Dizon, B. Pranggono, Journal of Ambient Intelligence and Humanized Computing (2021). DOI 10.1007/s12652-021-02970-y

7. G.W. Denardin, C.H. Barriquello, A. Campos, R.N.D. Prado. An intelligent system for street lighting monitoring and control (2009)

8. C. Iordache, S. Gavat, C. Mada, D. Stanciu, C. Holban, in IEEE International Conference on Automation, Quality and Testing, Robotics (AQTR 2008), IEEE International Conference on Automation, Quality and Testing, Robotics (AQTR 2008), vol. 3 (IEEE, ROMANIA, 2008), IEEE International Conference on Automation, Quality and Testing, Robotics (AQTR 2008), vol. 3

9. G. Jia, G. Han, A. Li, J. Du, IEEE Transactions on Industrial Informatics 14(11), 4995 (2018). DOI 10.1109/TII.2018.2857918

10. L. L., C. X., W. Y., W. Q. The development of road lighting intelligent control system based on wireless network control (2009)

11. S.R. Parekar, M.M. Dongre, in IEEE International Conference on Information Processing (ICIP) (IEEE, 2015), IEEE International Conference on Information Processing (ICIP), pp. 604-609

12. Y.S. Yang, S.H. Lee, G.S. Chen, C.S. Yang, Y.M. Huang, T.W. Hou, IEEE Access 8, 38568 (2020). DOI 10.1109/ACCESS.2020.2975708

13. A. Zanella, N. Bui, A. Castellani, L. Vangelista, M. Zorzi, IEEE Internet of Things Journal 1(1), 22 (2014). DOI 10.1109/JIOT.2014.2306328
14. F. Leccese, IEEE Transactions on Power Delivery 28(1), 21 (2013). DOI 10.1109/TPWRD.2012.2212215

15. F. Leccese, M. Cagnetti, D. Trinca, Sensors 14(12), 24408 (2014). DOI $10.3390 / \mathrm{s} 141224408$

16. F. Bellido-Outeiriño, F. Quiles-Latorre, C. Moreno-Moreno, J. Flores-Arias, I. Moreno-García, M. Ortiz-López, Sensors 16(5), 597 (2016). DOI 10.3390/s16050597

17. P.T. Daely, H.T. Reda, G.B. Satrya, J.W. Kim, S.Y. Shin, IEEE Sensors Journal 17(18), 6100 (2017). DOI 10.1109/JSEN.2017.2734101

18. P. Elejoste, I. Angulo, A. Perallos, A. Chertudi, I. Zuazola, A. Moreno, L. Azpilicueta, J. Astrain, F. Falcone, J. Villadangos, Sensors 13(5), 6492 (2013). DOI 10.3390/s130506492

19. A.Y. Hata, D. Habermann, F.S. Osorio, D.F. Wolf, in IEEE Intelligent Vehicles Symposium (IV) (IEEE, Dearborn, 2014), IEEE Intelligent Vehicles Symposium (IV), pp. 1319-1324

20. A. Garces-Jimenez, J.L. Castillo-Sequera, A. Del Corte-Valiente, J.M. Gomez-Pulido, E.P.D. Gonzalez-Seco, IEEE Access 7, 119881 (2019). DOI 10.1109/ACCESS.2019.2932055

21. S. Pizzuti, M. Annunziato, F. Moretti, Energy Efficiency 6(3), 607 (2013). DOI 10.1007/s12053-013-9195-9

22. J.F. De Paz, J. Bajo, S. Rodríguez, G. Villarrubia, J.M. Corchado, Information Sciences 372, 241 (2016). DOI 10.1016/j.ins.2016.08.045

23. P. Mohandas, J.S.A. Dhanaraj, X.Z. Gao, Sustainable Cities and Society 48, 101499 (2019). DOI 10.1016/j.scs.2019.101499

24. S. Escolar, J. Carretero, M.C. Marinescu, S. Chessa, International Journal of Distributed Sensor Networks 10(5), 971587 (2014). DOI 10.1155/2014/971587

25. J. He, Z. Zhu, F. Wang, J. Li, in International Conference on Intelligent Transportation, Big Data \& Smart City (ICITBS) (IEEE, CHINA, 2019), International Conference on Intelligent Transportation, Big Data \& Smart City (ICITBS), pp. 513-516

26. F. Yang, J. Xu, Asia \& the Pacific Policy Studies 5(3), 533 (2018). DOI 10.1002/app5.246

27. H.C. Lee, K.H. Ke, IEEE Transactions on Instrumentation and Measurement 67(9), 2177 (2018). DOI 10.1109/TIM.2018.2814082

28. U. Noreen, A. Bounceur, L. Clavier, in 3rd International Conference on Advanced Technologies for Signal and Image Processing (ATSIP) (IEEE, Fez, MOROCCO, 2017), 3rd International Conference on Advanced Technologies for Signal and Image Processing (ATSIP), pp. 1-6

29. J. Petäjäjärvi, K. Mikhaylov, M. Pettissalo, J. Janhunen, J. Iinatti, International Journal of Distributed Sensor Networks 13(3), 155014771769941 (2017). DOI 10.1177/1550147717699412

30. S. Chen, G. Xiong, J. Xu, S. Han, F.Y. Wang, K. Wang, in Chinese Automation Congress (CAC) (IEEE, CHINA, 2018), Chinese Automation Congress (CAC), pp. 1196-1200 